\title{
La necesaria revolución de la educación universitaria en el Perú
}

The necessary revolution of university education in Peru

Álvaro Adrián Rodríguez Barriga ${ }^{* *}$

\section{Resumen}

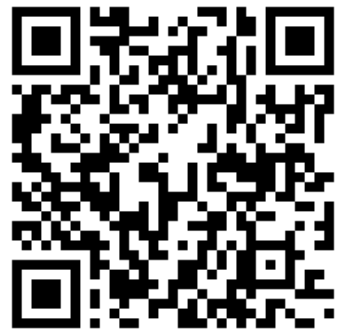

Un diagnóstico que nos permite atacar el problema de la universidad en el Perú incluye reconocer un inadecuado nivel académico en la formación universitaria, recursos económicos, nulos convenios entre universidades y ausentismo/desconexión entre el sector empresarial con universidad. Es claro que las iniciativas del estado hasta ahora han sido insuficientes y aun no se observan resultados dado que las reformas planteadas por el gobierno no sintonizan con las verdaderas necesidades de la sociedad. Además, los nuevos profesionales tienen que tener un perfil no solo capaz de ocupar un puesto laboral, sino, preparado para generar nuevos puestos de trabajo. Es imperativo cambiar antiguas metodologías de aprendizaje por métodos colaborativos y significativos, hacer de las universidades, centros de estudio donde se forme una sociedad reflexiva, crítica y consciente. Finalmente, es importante garantizar la preparación adecuada de los estudiantes en su época escolar y así acortar brechas académicas y cognitivas en su transición a la universidad.

\footnotetext{
* Artículo original derivado del Proyecto "Educación y su historicidad" Financiado por Universidad Católica de Santa María entre agosto 2018 y mayo 2019.

** Docente universitario, Universidad Católica de Santa María. Arequipa, Perú. Email: alvaro.rodriguez.arb@gmail.com. ORCID: https://orcid.org/0000-0003-4783016X
}

\section{Sinergias educativas}

Enero - junio Vol. 5 -1 - 2020

http://sinergiaseducativas.mx/index.php/revista/ elSSN: 2662-6661

revistasinergia@soyuo.mx

Recepción: 26 de septiembre 2018

Aprobación: 09 junio 2019

Pag 279-287

\section{Disponible en}

http://www.redalyc.org/articulo.oa?id=57356143200 21

Atribución/Reconocimiento-NoComercialCompartirlgual 4.0 Licencia Pública Internacional CC BY-NC-SA 4.0

https://creativecommons.org/licenses/by-ncsa/4.0/legalcode.es 
Palabras clave: problema de la universidad, necesidad de cambiar, metodologías, reformas, sociedad, eficacia, competitividad.

\begin{abstract}
A diagnosis that allows us to attack the problem of the university in Peru includes recognizing an inadequate academic level in university education, economic resources, null agreements between universities and absenteeism / disconnection between the business sector with university. It is clear that the initiatives of the peruvian state so far have been insufficient and results are not yet observed since the reforms proposed by the government do not tune into the true needs of society. In addition, new professionals must have a profile not only capable of occupying a job, but prepared to generate new jobs. It is imperative to change old learning methodologies for collaborative and meaningful methods, making universities into study centers where a reflexive, critical and conscious society is formed. Finally, it is important to ensure adequate preparation of students in their school age and thus shorten academic and cognitive gaps in their transition to university.
\end{abstract}

Key words: university problem, need to change, methodologies, reforms, society, effectiveness, competitiveness.

\title{
Introducción
}

Para el Consejo Nacional de Educación (2017), el objetivo estratégico 5 del PEN al 2021, propone asegurar una educación superior de calidad que consolide la formación integral de las personas y les permita desarrollar sus competencias, alcanzar niveles mayores de especialización y contribuir al desarrollo económico, social y cultural del país. Faltando 2 años para llegar a la meta, realmente ¿Estamos cerca de alcanzar dicho objetivo? ¿Cuán lejos estamos? ¿Estamos verdaderamente comprometidos a contribuir con una educación superior que encamine a nuestros jóvenes a que contribuyan al desarrollo del país?

Considero que la raíz del problema incide fundamentalmente en la falta de visión de las universidades que no tiene como centro de sus 
actividades a los estudiantes, la falta de conciencia por una formación de calidad (Cevallos, 2014), fallas del sistema educativo básico regular que se arrastran hasta la universidad y la manera tradicional de enseñanza y antiguas metodologías que lamentablemente siguen vigentes en las universidades del Perú a pesar de estar inmersos en constantes procesos de evaluación y acreditación. Ante este panorama podemos decir entonces que estamos en un proceso de globalización de la educación que se entiende como esfuerzos para lograr que los estudiantes reciban contenidos utilizando las nuevas tecnologías, es decir, utilizar la tecnología y entornos digitales como un medio para potenciar habilidades experiencias y capacidades que reconozcan a los estudiantes como dueños del conocimiento que recibieron y estar listos para usarlo en sus diversos contextos, es decir, "Saber hacer".

Este artículo abordará los principales problemas por los que atraviesa el sistema educativo superior en el Perú, repasando el papel fundamental que cumplen los estudiantes, cuerpo docente, órganos administrativos y estado para empezar a desarrollar una nueva visión de la formación profesional encaminada a desarrollar jóvenes listos a afrontar los verdaderos desafíos de nuestra época.

Es importante revisar y mencionar fundamentos de la educación superior en el Perú para una adecuada y sólida sustentación de la presente investigación. De acuerdo a las leyes vigentes en el país, (1992, Ley de la Educación Universitaria $N^{0}$ 23733) son fines de la universidad peruana: 1 . Conservar, acrecentar y transmitir la cultura universal con sentido crítico y creativo afirmando preferentemente los valores nacionales. 2. Realizar investigación en las humanidades, las ciencias y las tecnologías, y fomentar la creación intelectual y artística. 3. Extender su acción y sus servicios a la comunidad, y promover su desarrollo integral.

Justamente es el punto 3 donde se genera la principal controversia y es motivo de la elaboración de este artículo ya que como he expuesto anteriormente no se percibe que la universidad realmente esté desarrollando INTEGRALMENTE a sus estudiantes. 


\section{Materiales y métodos}

La investigación corresponde al campo cualitativo. Para la elaboración de este estudio se utilizó principalmente la revisión bibliográfica de diversos estudios sobre la realidad de la universidad en el Perú como son "Informe Bienal sobre la realidad universitaria en el Perú, 2018” y "Proyecto Educativo Nacional al 2021".

\section{Resultados}

\section{A. ENCONTRANDO PROBLEMAS}

De acuerdo al (Informe Bienal sobre la Realidad Universitaria, 2018) he podido distinguir 5 grandes categorías que pintan de cuerpo entero la problemática de la universidad; el inadecuado nivel académico, economía, investigaciones de baja calidad, nulos convenios entre universidades y ausentismo del sector empresarial para potencias las habilidades de los estudiantes.

Como ya se adelantó, se pretende centrar los esfuerzos en el problema que se considera más grave desde el punto de vista de esta investigación que es el inadecuado nivel académico de la universidad en el Perú (Informe sobre educación superior universitaria, 2002). Lamentablemente, en nuestro país hay una concepción errónea de que la universidad es la obligada continuación del colegio, lo que trae como consecuencia, que la deficiente preparación impartida en los últimos años del colegio se arrastre a la universidad (Cuenca, 2015). no hace otra cosa que arrastrar el lastre de la mala preparación impartida en la escuela secundaria.

Asimismo, otro problema que merece obligada atención y una necesaria revolución es la falta de movilidad académica, intercambios y colaboración académica entre universidades. Esta problemática se evidencia con claridad en el cuerpo docente de las casas universitarias, quienes sólo se contentan con tener carga académica dejando de lado la contribución científica entre docentes de escuelas y facultades a fin de diversas casas de estudio. La notable desconexión que existe entre el sector productivo del país y los recién graduados o estudiantes de últimos años afecta en gran forma 
el desarrollo integral de los universitarios ya que no se les permite una orgánica ni rápida inserción al mercado laboral para pasar a ser parte del sector productivo del Perú. Anthony Middlebrook, vicepresidente de Recursos Humanos de Alicorp en el CADE 2013, indicó que los empresarios tienen que hacer una gran inversión para insertar a los nuevos profesionales a sus filas.

Lamentablemente un problema estructural que merece especial atención de entes gubernamentales, donde se garantice la accesibilidad al sistema universitario. Sólo 3 de cada 10 jóvenes pueden estudiar en una universidad en el Perú. El estado tiene que estar en la capacidad de generar adecuadas políticas encaminadas a la inclusión y accesibilidad de estudios superiores, teniendo como aliado estratégico a las nuevas tecnologías de información.

\section{B. ¿LISTOS PARA AFRONTAR NUEVOS RETOS?}

¿En realidad se están formado profesionales que la sociedad necesita y listos para afrontar los nuevos retos de la globalización? La formación universitaria en nuestro país está desalineada con esa premisa dado que las casas de estudio superiores en primera instancia no realizan un diagnóstico de las verdaderas necesidades del país y en una segunda instancia se puede observar una clara formación tradicional muy enmarcada en conocimiento netamente teóricos dejando de lado muchas veces el trabajo práctico y colaborativo entre estudiantes. Se tiene que empezar a formar profesionales que no solo sean simples empleados de grandes o medianas corporaciones dispuestos a cumplir jornadas laborales, sino a profesionales capaces de resolver problemas con un enfoque consciente de su entorno, reflexivo y crítico.

Ante este problema, ¿Dónde queda y cuál debe ser el aporte del estado? Esta investigación postula que las universidades deben recibir un aporte económico y político por parte del estado para cubrir necesidades constantes y necesarias como la mejora de sueldos para docentes, implementación logística de sus instalaciones, mejora de accesibilidad, capacitación, etcétera.

\section{LA NECESARIA REVOLUCIÓN: PASO POR PASO}


El primer y necesario paso para revolucionar la educación superior en el Perú es mejorar las condiciones básicas bajo las que opera la universidad. No es posible exigir altos estándares de calidad ni grandes resultados si las universidades no cuentan con recursos ni políticas que les permita un correcto desenvolvimiento de sus funciones básicas de formación. Es importante incidir también en mecanismos que permitan monitorear la disposición de los recursos para que su direccionamiento esté encaminado de manera eficiente. Si bien, el Estado no va a estar en la capacidad de poder fomentar recursos a todas las universidades, si es posible que este actúe como gestor ante entes internacionales.

El segundo paso necesario es trabajar el ADN de las universidades, tal como señalaba De Sousa, trabajar en la "hegemonía" de la universidad es necesario para que los fines que persiga queden claros y no se confundan con otras funciones que no le correspondan. En el caso del Perú, la universidad tiene que estar orientada a formar ciudadanos plenos, donde el aprendizaje debe estar garantizado en entornos que le permitan al estudiante sentir, actuar y aprender partiendo de experiencias tanto buenas como malas, ya que es lo más cercano y real que tendrá en su entorno profesional.

Ante esta idea, es necesario, criticar el hecho que en la universidad tradicional siempre se ha premiado a las mejores notas, a la respuesta "correcta", pero el error siempre es castigado y no es visto como una oportunidad de mejora. El educador Santiago Moll señala la importancia del error en el aprendizaje. Moll refiere que un alumno que jamás se equivoca nunca aprenderá nada nuevo. Además, premiando el error se genera confianza, autoestima y autoconcepto. ¿Será entonces que el método actual bajo el que opera la universidad hace que los alumnos teman equivocarse? Estamos perdiendo valiosas oportunidades de aprendizaje autónomo y significativo. ¿ $\mathrm{Si}$ no permitimos que los estudiantes se equivoquen en la universidad, en qué otro momento podrán permitirse hacerlo?

Cuando De Sousa manifiesta una "crisis institucional" en el caso de la problemática de la universidad colombiana, revisé que es una problemática que también se debe abordar para el caso peruano. Actualmente, todas las universidades están en la mira de SUNEDU, tratando de cumplir todos los estándares que se exige para cumplir 
con el licenciamiento y continuar operando en el marco que la ley exige. Sin embargo, al ocupar esfuerzos en cumplir con requerimientos, se descuida ampliamente otro aspecto importante que es la definición de objetivos y valores que persigue la universidad en el marco de su formación profesional. ¿Acaso solo se está cumpliendo con el papeleo para el momento? ¿No es acaso una visión demasiado cortoplacista?

Con este análisis no quiero decir que estoy en contra de la fiscalización que hace la SUNEDU, sino que los parámetros de calidad que exige deberían enfocarse más en la persona, en el estudiante y no en aspectos administrativos.

El tercer paso está relacionado a trabajar en medidas necesarias a mediano y largo plazo. No se puede ser cortoplacista si el objetivo es crear mejores condiciones en la universidad. Para ello, la visión debe ser sistémica para la intervención del problema, es decir, no atacar necesariamente el problema aparente sino intervenir en alguna de las raíces del problema que como ya se estableció previamente es la preparación adecuada de los estudiantes en su época escolar y así acortar brechas, muchas veces, difíciles de superar.

Además, junto a esta visión debe plantearse de manera imprescindible retomar el camino y papel que tiene la universidad peruana en el desarrollo de nuestro país, siendo una aliada para la solución multidisciplinaria de los problemas que aquejan al país considerando a la patria dentro del proceso continuo de globalización.

Quizás, la pregunta a esta altura de este artículo es, ¿Quién debería liderar esta revolución? Considero que debe ser un trabajo que comprometa en primer orden a la universidad apoyada por el estado que debe garantizar condiciones básicas para empezar a retomar el camino y construir una nueva universidad nueva mucho más democrática considerando la globalización constante.

Quedan entonces tareas pendientes, quizás comenzar con el cambio debe estar relacionado a conectar a la universidad como una suerte de "sociedad universitaria abierta", donde se propicien espacios para investigar y discutir con una óptica crítica hacia los problemas que 
realmente necesitan ser resueltos. Sin embargo, para ejercer una crítica real y formativa, la universidad debe ser libre de ataduras administrativas y esquemas académicos que lamentablemente aún permanecen hoy en día, tal como lo planté anteriormente. Estos espacios podrían generar una nueva universidad que no solo esté orientada a formar nuevos profesionales sino a formar personas capaces de reflexionar sobre las necesidades que necesitan ser cubiertas en sus entornos a través de sus competencias y conocimientos que surgen en los mismos espacios de la comunidad universitaria abierta.

\section{Discusión}

Para afrontar un verdadero proceso de cambio radical de la educación universitaria en el país se deben reconocer los siguientes problemas: inadecuado nivel académico, incertidumbre económica, nulos convenios entre universidades y ausentismo/desconexión entre el sector empresarial con universidad.

Las universidades deben enfocar esfuerzos por formar profesionales que la sociedad realmente necesita mediante nuevas metodologías de aprendizaje que dejen de lado el enfoque teórico para dar paso a una metodología que incluya la práctica con el trabajo colaborativo y significativo.

Los nuevos profesionales producto de la revolución de la educación universitaria propuesta en esta investigación tienen que ser personas que no solamente estén dispuestos a cumplir jornadas laborales sino con plenas capacidades para generar empresas y fomentar nuevas oportunidades laborales.

Mejorar las condiciones económicas de las universidades es imperativo para que las casas de estudio puedan permitirse un correcto desenvolvimiento de sus funciones básicas de formación. Asimismo, es necesario introducir en los cimientos de las universidades premisas orientadas a ser instituciones eficaces $y$ competitivas.

Introducir la visión sistémica en la intervención del problema de la universidad en el Perú para no atacar solo a las consecuencias del 
problema sino a la raíz del mismo, es decir, garantizar la preparación adecuada de los estudiantes en su época escolar y así acortar brechas académicas y cognitivas en su transición a la universidad.

Si realmente estamos comprometidos en cambiar la universidad también estaremos implicados en la búsqueda de una nueva sociedad pensante, con capacidad reflexiva, democrática y crítica. La universidad peruana de HOY no permite construir una sociedad con esas características. La ansiada revolución de la educación universitaria está en nuestras manos.

\section{Referencias}

Cevallos, D. (2014). La calidad educativa en la realidad universitaria peruana frente al contexto latinoamericano. Flumen, 7(1), 3-8. Recuperado de http://repositorio.usat.edu.pe/handle/usat/104

Cuenca, Ricardo. (2015). La educación universitaria en el Perú: Democracia, expansión y desigualdades. Lima, Perú: Instituto de Estudios peruanos.

Superintendencia Nacional de Educación Superior Universitaria. (2018). Informe Bienal sobre la realidad universitaria 2014 - 2017. Recuperado de: https://www.sunedu.gob.pe/informe-bienal-sobrela-realidad-universitaria-guias-formatos/

Congreso de la República de Perú. (1992). Ley de la Educación Universitaria $N^{o} \quad 23733 . \quad$ Recuperado de: https://www.albany.edu/dept/eaps/prophe/data/Country_Law/PeruLEY\%20UNIVERSITARIA_modificada.pdf

Consejo Nacional de Educación, Ministerio de Educación. (2007). Proyecto Educativo Nacional al 2021. Recuperado de: http://www.cne.gob.pe/proyecto-educativo-nacional/

Sousa Santos, B.(2007). La universidad en el siglo XXI. Bolivia: Plural Editores 